\title{
Les regalia du président
}

Autour des cadeaux diplomatiques ivoiriens de Valéry Giscard d'Estaing The president's regalia. On Valéry Giscard d'Estaing's Ivorian diplomatic gifts

\section{Alexandre Girard-Muscagorry}

\section{Q OpenEdition}

1 Journals

Édition électronique

URL : http://journals.openedition.org/cel/840

DOI : $10.4000 / \mathrm{cel} .840$

ISSN : 2262-208X

Éditeur

École du Louvre

\section{Référence électronique}

Alexandre Girard-Muscagorry, "Les regalia du président », Les Cahiers de l'École du Louvre [En ligne], 12 | 2018, mis en ligne le 03 mai 2018, consulté le 17 septembre 2019. URL : http:// journals.openedition.org/cel/840; DOI : 10.4000/cel.840

Ce document a été généré automatiquement le 17 septembre 2019.

\section{(c) (i) () $\Theta$}

Les Cahiers de l'École du Louvre sont mis à disposition selon les termes de la licence Creative Commons Attribution - Pas d'Utilisation Commerciale - Pas de Modification 4.0 International. 


\title{
Les regalia du président
}

\author{
Autour des cadeaux diplomatiques ivoiriens de Valéry Giscard d'Estaing \\ The president's regalia. On Valéry Giscard d'Estaing's Ivorian diplomatic gifts
}

\section{Alexandre Girard-Muscagorry}

1 Du 11 au 15 janvier 1978, Valéry Giscard d'Estaing, alors président de la République française, se rend en voyage officiel en Côte d'Ivoire, à la rencontre de son homologue, Félix Houphouët-Boigny. À la suite du Gabon et du Zaïre (5-9 août 1975), du Mali (13-15 février 1977) et du Sénégal (19-23 avril 1977), ce quatrième voyage officiel en direction du "premier carré des "amis de la France" " constitue un déplacement diplomatique des plus classiques, tant du point de vue de la destination choisie que de l'organisation générale du séjour. En collaboration avec le protocole ivoirien et l'ambassade de France, les services de la présidence française ont, en effet, soigneusement préparé le programme de cette semaine, articulé autour de cérémonies protocolaires (dîner officiel, discours à l'hôtel de ville, réception à l'ambassade française), de visites culturelles et d'entretiens bilatéraux. Au terme de deux jours à Abidjan, Valéry Giscard d'Estaing est invité à se rendre à Yamoussoukro, village natal d'Houphouët-Boigny, transformé sous son impulsion en centre urbain, politique et économique majeur au cours des années 1960-1970². Bien inscrite au programme de la visite, l'« arrivée à la Mairie avec allocutions du Député-Maire, du Porte-Parole des Chefs coutumiers et du Président de la République française ${ }^{3}$ » n'en demeure pas moins surprenante dans le contexte très encadré, voire normalisé, des réceptions officielles. À la suite d'un discours consensuel sur le «rôle irremplaçable joué par la France dans le processus de développement ${ }^{4}$ ", Valéry Giscard d'Estaing est en effet élevé, sous le regard d'un aréopage d'élites locales, à la dignité de «Président honoraire de l'“Association des chefs coutumiers" ${ }^{\prime}$. En guise de marqueur de son intronisation, le chef d'État français reçoit plusieurs objets en or et en bois doré, associés à l'exercice du pouvoir en pays akan : un trône, une couronne, une paire de sandales, deux pendentifs, un sabre d'apparat, un chasse-mouches ainsi que trois cannes dites « de linguistes». Si les objets sont offerts au nom des autorités coutumières de Yamoussoukro, les clichés réalisés lors de la cérémonie par le service photographique de l'Élysée montrent Félix Houphouët-Boigny en train de présenter et de commenter les divers insignes disposés sur une table, laissant penser qu'il 
fut sinon à l'initiative de ces présents, du moins informé en amont de la nature des cadeaux offerts (fig. 1).

Fig. 1

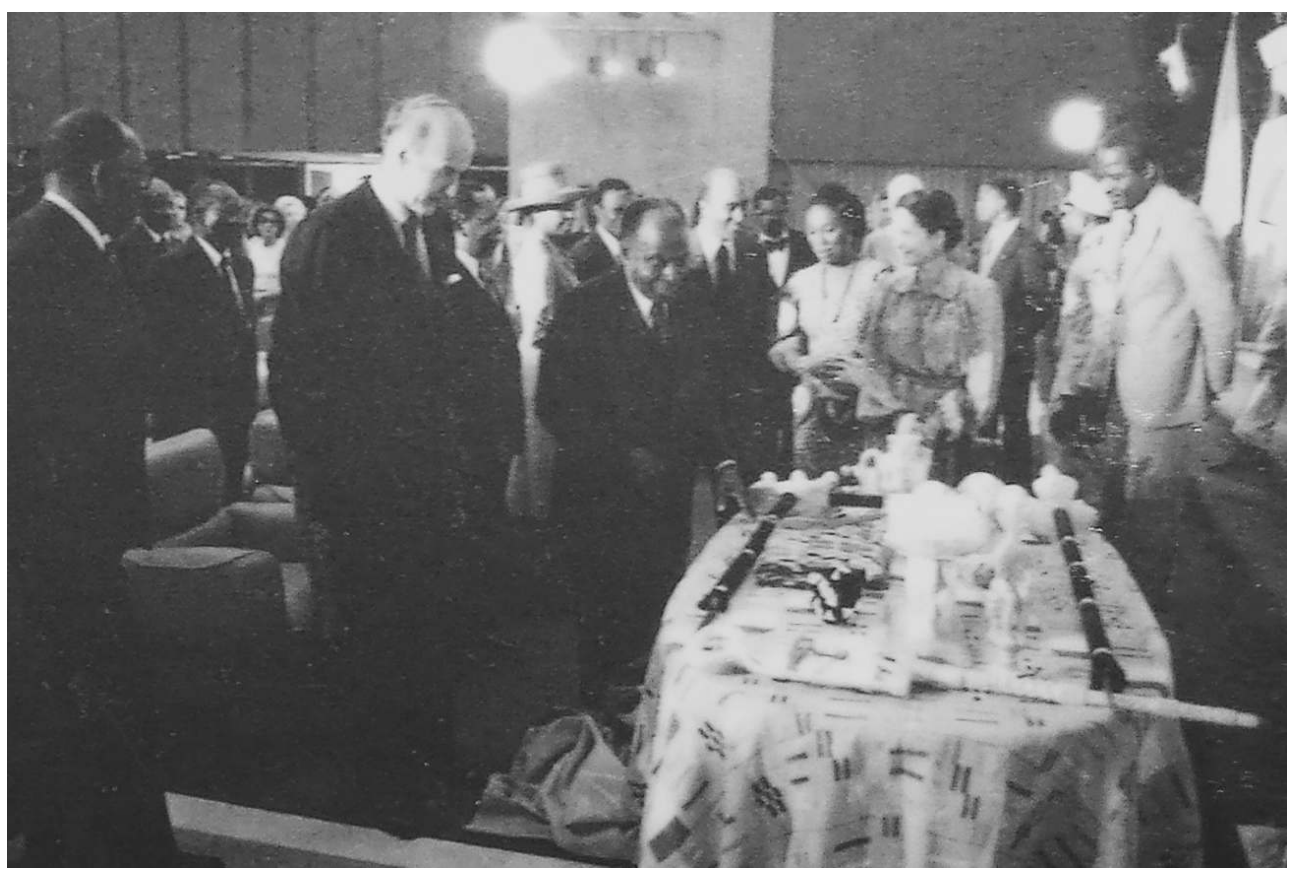

Voyage officiel de Valéry Giscard d'Estaing en Côte d'Ivoire, cérémonie à l'hôtel de ville de Yamoussoukro, 11-15 janvier 1978, Archives nationales, AG/5(3)/3506, reportage n 3733 (détail)

(c) photographie de l'auteur

2 Aujourd'hui conservées dans les collections du musée du quai Branly - Jacques Chirac ${ }^{6}$, ces pièces ne peuvent manquer d'interpeller : outre l'intérêt plastique des objets, bien éloignés des présents standardisés - sculptures d'animaux sauvages ou de «types ethniques ", peintures de paysages pittoresques - offerts en grand nombre par les chefs d'État africains, leur forte symbolique royale s'oppose en apparence à la dimension républicaine des charges politiques dont le donateur et le donataire sont investis. Le choix de ces objets est pourtant loin d'être anecdotique. Souvent perçus comme de simples pièces de l'«attirail » diplomatique, les cadeaux protocolaires font, en réalité, l'objet de processus de sélection complexes, prenant en compte à la fois le statut voire la personnalité du donateur et des enjeux de représentation nationaux plus vastes ${ }^{7}$. Leur histoire demeure néanmoins délicate à retracer, compte tenu de sources éclatées ou parcellaires. À l'inverse, les cadeaux ivoiriens de Valéry Giscard d'Estaing présentent l'originalité d'avoir suscité un nombre significatif de commentaires, formulés tant par le président que par la presse ou l'institution muséale qui en fut dépositaire, et de s'inscrire dans un contexte de remise bien documenté par les sources archivistiques françaises. Dans la veine des travaux de Christaud M. Geary ${ }^{8}$ ou de Mary Jo Arnoldi ${ }^{9}$, attachés à restituer le parcours méandreux de présents protocolaires africains aujourd'hui conservés au National Museum of African Art, cet article se propose de mettre en lumière les différentes significations prises par ces insignes de Côte d'Ivoire dans le contexte d'un échange diplomatique et de souligner la façon dont leur qualification, «au double sens d'identification et d'attribution de valeur ${ }^{10}$ ", évolua en France sous l'influence du contexte institutionnel et politique de l'époque. 


\section{"Les objets en bois doré qui symbolisent le pouvoir »}

3 À l'exception du terme "akan ", l'origine exacte des objets en or offerts au chef d'État français reste mystérieuse. C'est en effet sous cet ethnonyme que les cadeaux furent présentés le 28 juin 1978 devant le Comité technique du musée des Arts africains et océaniens, alors chargé de statuer sur la pertinence de la libéralité consentie par le président au profit de l'institution muséale ${ }^{11}$ : «Fabriqués pour la circonstance à Abengourou par des artisans ghanéens, ils constituent les attributs traditionnels des chefs coutumiers $a_{k a n}{ }^{12}$.» Cette attribution du travail à des orfèvres et sculpteurs ghanéens installés en territoire ivoirien aurait été suggérée à Étienne Féau, rattaché à la conservation du musée, par un certain François Dubois ${ }^{13}$. Cependant, de l'avis de Bohumil Holas, conservateur du musée d'Abidjan et auteur de plusieurs ouvrages sur les arts de la Côte d'Ivoire, l'origine des objets apparaît plus complexe. Si les sandales proviennent certainement de l'est du pays, d'Abengourou ou de Bondoukou, de même que le trône de " type anyi ", l'un des pendentifs en forme de crocodile bicéphale s'apparente plutôt aux productions contemporaines de Blokosso, un village ébrié des faubourgs d'Abidjan ${ }^{14}$.

4 En dépit des incertitudes concernant leur contexte de production, ces cadeaux entendent constituer des exemples emblématiques de l'usage des objets en or chez les populations du centre et du sud-est de la Côte d'Ivoire. Baoulé, Ébrié, Anyi sont autant d'ethnonymes qui renvoient à l'ensemble plus large des populations akan, présentes sur les territoires actuels du Ghana et de la Côte d'Ivoire. Les Ashanti du Ghana sont particulièrement renommés pour la production d'insignes de pouvoir en or utilisés aux différents échelons de la hiérarchie coutumière. Si les populations akan de la Côte d'Ivoire produisent également des ornements personnels en or, ceux-ci font en comparaison l'objet d'une moindre utilisation et apparaissent moins sophistiqués. Timothy Garrard, à partir de l'exemple baoulé, explique ce décalage par une centralisation politique moins affirmée sur le territoire ivoirien ou, tout au moins, plus récente ${ }^{15}$. À l'époque précoloniale, les Baoulé ne disposaient pas de chefs traditionnels d'un rang supérieur aux anciens des villages, à l'exception de la région de Sakassou où des migrants du Ghana avaient introduit le modèle de la chefferie ghanéenne. L'administration coloniale française, souhaitant consolider son pouvoir sur les populations installées au centre et au sud de la colonie à travers la création de chefs de district, contribua toutefois à renforcer le rôle de certaines figures d'autorité locales. Ces dernières cherchèrent à asseoir leur statut par l'adoption de regalia de style akan. L'usage de bracelets, de bagues, de sandales dorées, de chaînes, etc., souvent directement achetés à des orfèvres ashanti de Kumasi (Ghana), fut alors popularisé au sein des élites coutumières akan de Côte d'Ivoire désireuses de marquer leur nouveau statut ${ }^{16}$. Selon les informations d'Étienne Féau, les regalia offerts à Valéry Giscard d'Estaing sont donc directement issus des circuits d'approvisionnement qu'empruntent habituellement les objets destinés aux chefferies ivoiriennes.

De surcroit, ces objets forment, du point de vue typologique et iconographique, un ensemble représentatif des insignes de pouvoir akan. Sans nous appesantir sur l'usage et les significations de chaque objet, il convient de souligner la forte charge symbolique de ces pièces. Les sandales (fig. 2), en premier lieu, sont un des principaux marqueurs de rang car le chef, considéré comme un être d'essence divine, ne peut en principe pas toucher la terre de ses pieds nus au risque de provoquer une famine ${ }^{17}$. De même, si les couronnes, qui adoptent ici la forme de bandeaux de velours sur lesquels sont rapportés 
des éléments figuratifs ou géométriques en bois plaqué de feuilles d'or, n'ont pas une importance aussi forte que les sandales, elles témoignent cependant du haut rang du porteur ${ }^{18}$ (fig. 3). Les trois cannes de «linguiste» ou de "porte-parole» sont, quant à elles, généralement tenues par l'okyeame, principal conseiller et porte-parole du chef. Les sculptures figuratives en ronde-bosse qui ornent leur extrémité constituent des messages proverbiaux, le plus souvent en rapport avec le pouvoir du chef et la continuité de la chefferie $^{19}$. Une des cannes reçues par le chef d'État français représente ainsi deux hommes assis sur des tabourets, se serrant la main sous un grand parasol, probable allusion à la rencontre bilatérale entre Félix Houphouët-Boigny et Valéry Giscard d'Estaing (fig. 4).

Fig. 2

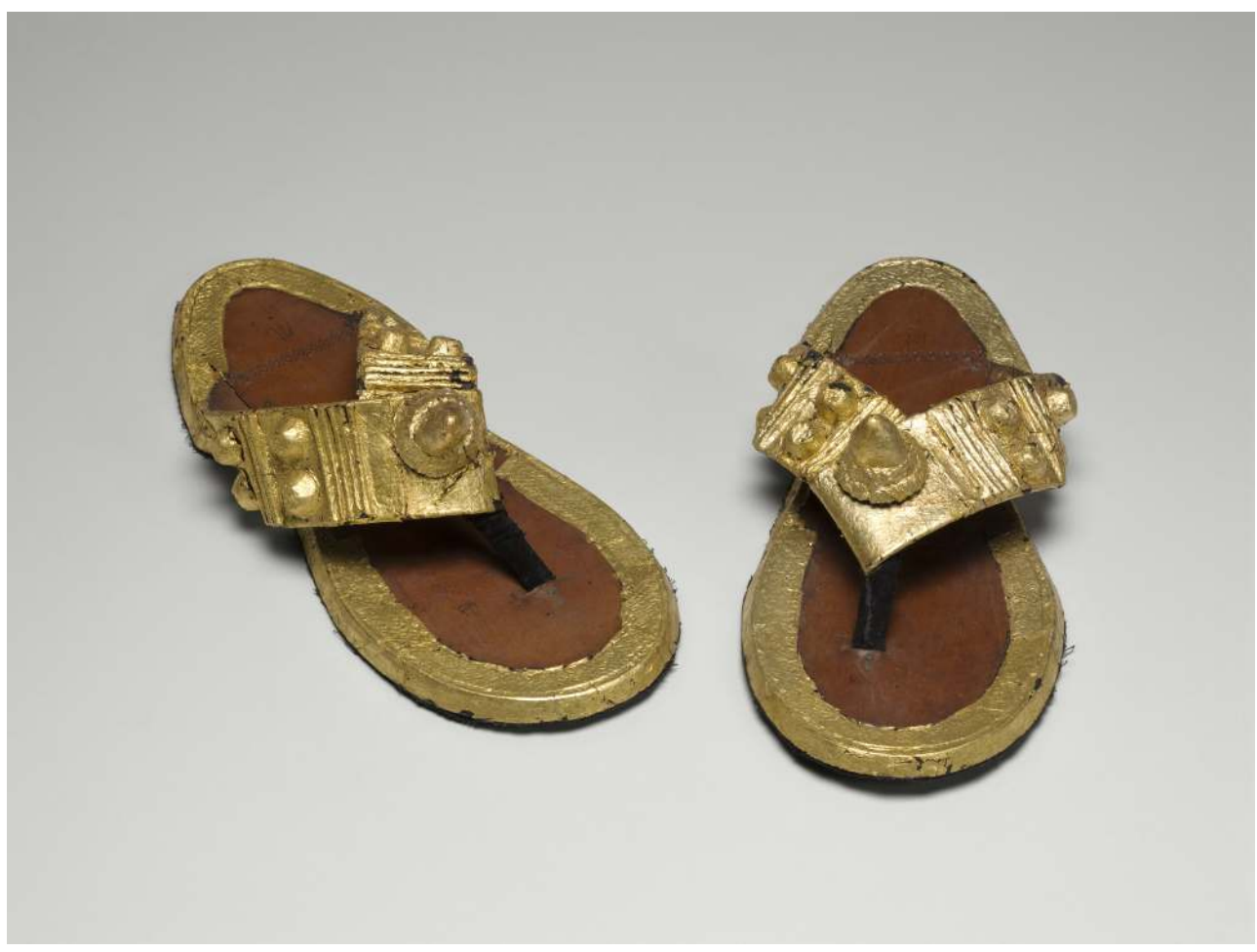

Paire de sandales, cuir, feuilles d'or, L. 29 cm ; I. 12 cm, Paris, musée du quai Branly - Jacques Chirac, inv. $n^{\circ}$ 73.1978.2.8.1-2

(c) musée du quai Branly - Jacques Chirac, photo Thierry Ollivier, Michel Urtadoc 
Fig. 3

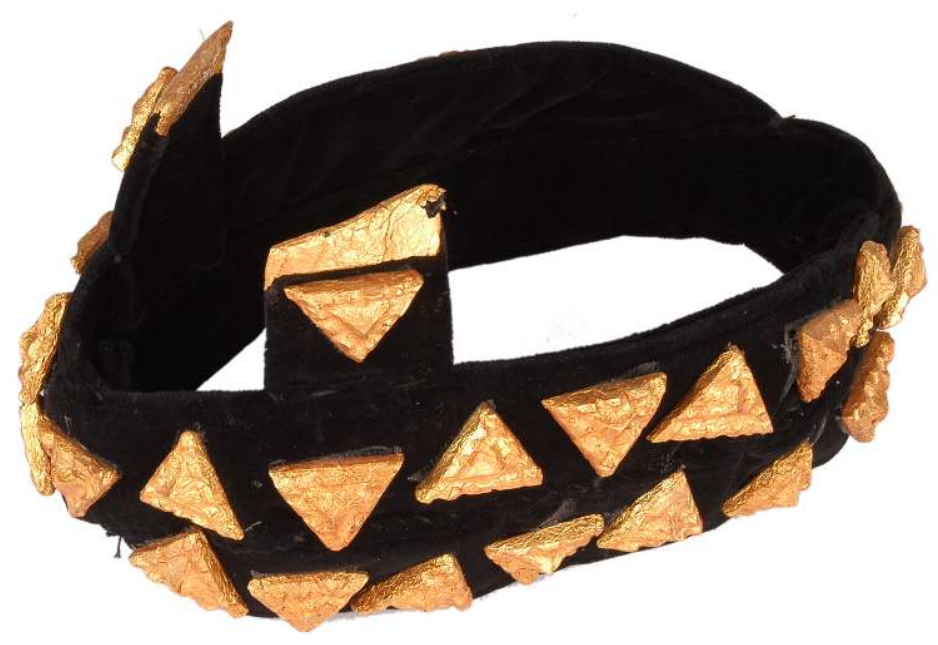

Bandeau, bois, feuilles d'or, velours, Diam. 20 cm ; H. 12,5 cm, Paris, musée du quai Branly - Jacques Chirac, inv. n 73.1978.2.7

(C) musée du quai Branly - Jacques Chirac

6 Bien différents des «souvenirs " ivoiriens standardisés, les cadeaux remis à Valéry Giscard d'Estaing constituent des objets de prix à la facture soignée, semblables aux regalia en usage dans les chefferies akan de Côte d'Ivoire. Leur statut d'objets de commande, ainsi que semble l'indiquer la canne à l'effigie des deux présidents, n'ampute nullement le fort symbolisme sous-jacent, en lien avec l'expression de la souveraineté chez les populations akan. Le caractère hautement honorifique de ces insignes fut d'ailleurs bien perçu par Valéry Giscard d'Estaing, qui accorda une place toute particulière à la remise de ces cadeaux dans ses mémoires : " Nous avons fait un premier arrêt à la mairie de Yamoussoukro où j'ai été intronisé chef coutumier de Côte d'Ivoire et où on m'a remis les objets en bois doré qui symbolisent le pouvoir ${ }^{20}$. " Cependant, peut-on limiter notre appréhension de ces objets à celle d'un simple hommage rendu à la dignité du chef d'État français? 
Fig. 4

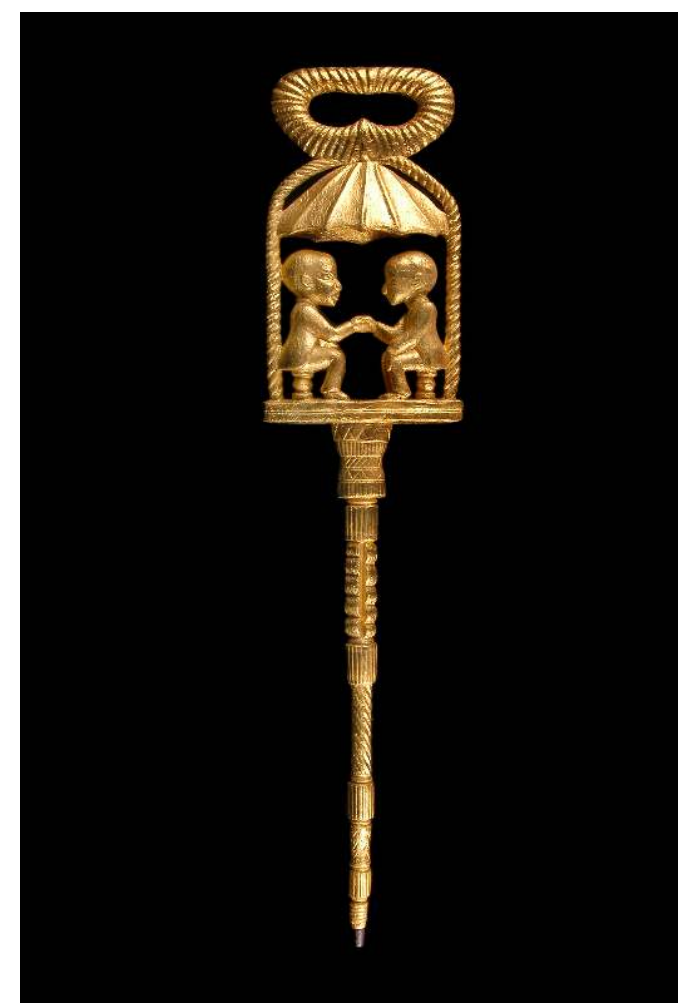

Canne figurative, bois, feuilles d'or, H. $104 \mathrm{~cm} ; 1.23,3$ cm, Paris, musée du quai Branly - Jacques Chirac, inv. no 73.1978.2.1

(c) musée du quai Branly - Jacques Chirac

\section{Félix Houphouët-Boigny, « descendant d'une ancienne et riche famille de chefs traditionnels de la tribu Baoulé, célèbre pour son art »}

7 Outre la nécessité d'accorder la valeur du présent au statut du destinataire, les cadeaux diplomatiques apparaissent souvent pour les donateurs comme un moyen privilégié d'évoquer leur histoire personnelle, leur parcours politique ou leurs goûts artistiques. François Mitterrand, connu comme un fin bibliophile, remettait volontiers des ouvrages d'auteurs français à ses invités ou hôtes de marque. De même Jacques Chirac s'attachera$\mathrm{t}$-il, après l'ouverture du Pavillon des Sessions en 2001 - l'espace dédié aux «arts premiers » au sein du musée du Louvre -, à offrir fréquemment le catalogue Sculptures de l'exposition, soulignant ainsi en filigrane son vif intérêt pour les civilisations extraoccidentales. La nature des présents reçus par Valéry Giscard d'Estaing invite dès lors à les relier à l'histoire personnelle du président ivoirien. En marge de sa carrière au sein des institutions politiques coloniales puis ivoiriennes, Félix Houphouët-Boigny fut également chef baoulé par le lignage de sa mère et ne cacha pas cette ascendance royale. Son autobiographie, Mes premiers combats, consacre notamment un long développement à l'origine historique des Baoulé et leur appartenance à l'ensemble akan, ainsi qu'à la descendance de sa propre famille ${ }^{21}$. Les archives protocolaires de l'Élysée témoignent, par ailleurs, de la diffusion de ce récit au-delà des frontières ivoiriennes. Le dossier 
préparatoire au déplacement présidentiel contient, par exemple, une « note biographique de Monsieur Félix Houphouët-Boigny» qui mentionne explicitement les origines du président ivoirien, « descendant d'une ancienne et riche famille de chefs traditionnels de la tribu Baoulé, célèbre pour son $\operatorname{art}^{22}$ ». Offrir ces regalia akan à un président français dont il était proche politiquement et personnellement apparaissait certainement comme un moyen de rendre hommage et de valoriser son histoire personnelle. Du moins est-ce ainsi que Valéry Giscard d'Estaing semble avoir interprété les cadeaux reçus à Yamoussoukro et, plus largement, ce déplacement sur les terres de son homologue. "J'étais impatient, écrit-il, de me rendre dans ce qu'il appelle "mon village", son lieu de naissance, Yamoussoukro, pour essayer de remonter la filière de ses origines ${ }^{23}$. » Plus loin, c'est la vision fantasmatique de l'expérience du pouvoir coutumier par le jeune Houphouët-Boigny qu'il convoque :

J'imagine le petit garçon qu'était alors Houphouët-Boigny, ployant sous ses vêtements chamarrés, portant la coiffure du chef, et tenant avec effort le bâton de commandement dont l'extrémité est décorée de trois lions recouverts d'une feuille d'or - le même dont on m'a remis une reproduction tout à l'heure à l'hôtel de ville de Yamoussoukro -, le petit garçon aux yeux écarquillés devant le spectacle des esclaves ivres et du sorcier qui attend la fin de la cérémonie, le sabre au fil tranchant posé sur les genoux ${ }^{24}$.

8 Malgré les erreurs d'interprétation - l'objet évoqué n'est pas un «bâton de commandement » mais une canne de linguiste - et l'exotisme de la plume du président français, ce passage montre que les présents reçus par ce dernier à Yamoussoukro atteignirent l'un de leurs objectifs : réveiller dans l'esprit du donataire le souvenir de la noble extraction de Félix Houphouët-Boigny.

\section{La récupération nationale des symboles akan}

De fait, les regalia akan offerts à Valéry Giscard d'Estaing ne font pas figure d'exception dans le corpus des cadeaux offerts par Félix Houphouët-Boigny. Le 21 mai 1982, François Mitterrand, également de passage à Yamoussoukro lors d'un voyage officiel en Côte d'Ivoire (17-24 mai 1982), reçut un ensemble similaire d'insignes de pouvoir des mains du président ivoirien, comprenant un tabouret, une couronne, un sabre et un chassemouches ${ }^{25}$. La remise des présents eut lieu dans le même cadre, l'hôtel de ville, et selon un dispositif analogue, marqué toutefois par un sens de la mise en scène plus affirmé, comme l'atteste le reportage du service photographique de l'Élysée ${ }^{26}$ : François Mitterrand, debout sur la tribune, reçoit le sabre et un bâton de linguiste, encadré à l'arrière-plan par deux femmes présentant les chasse-mouches. Ces présents furent complétés, par ailleurs, par un portrait du président français en chef coutumier, drapé dans un tissu kente, coiffé d'un calot de velours orné d'appliques et arborant un pendentif en or sur sa poitrine ${ }^{27}$ (fig. 5). Outre les insignes de pouvoir, il est intéressant de noter que Félix HouphouëtBoigny offrit, à plusieurs reprises, des productions matérielles présentées par le protocole comme baoulé. Ainsi, à l'occasion de sa première visite d'État aux États-Unis, très largement médiatisée en Côte d'Ivoire comme aux États-Unis ${ }^{28}$, le président ivoirien entendait remettre à John F. Kennedy " a rare Baoule (tribal) mask », comme l'indique une note du chef de protocole américain ${ }^{29}$. Faut-il voir dans cette promotion au rang de cadeau diplomatique d'objets archétypaux des productions akan et, singulièrement, baoulé, un attachement personnel du chef d'État ivoirien au patrimoine matériel de cette aire culturelle? Rappelons ici que Félix Houphouët-Boigny, bien connu comme amateur 
de mobilier de style, aurait aussi collectionné de l'art africain, dont un certain nombre d'ornements akan en or ${ }^{30}$.

Fig. 5

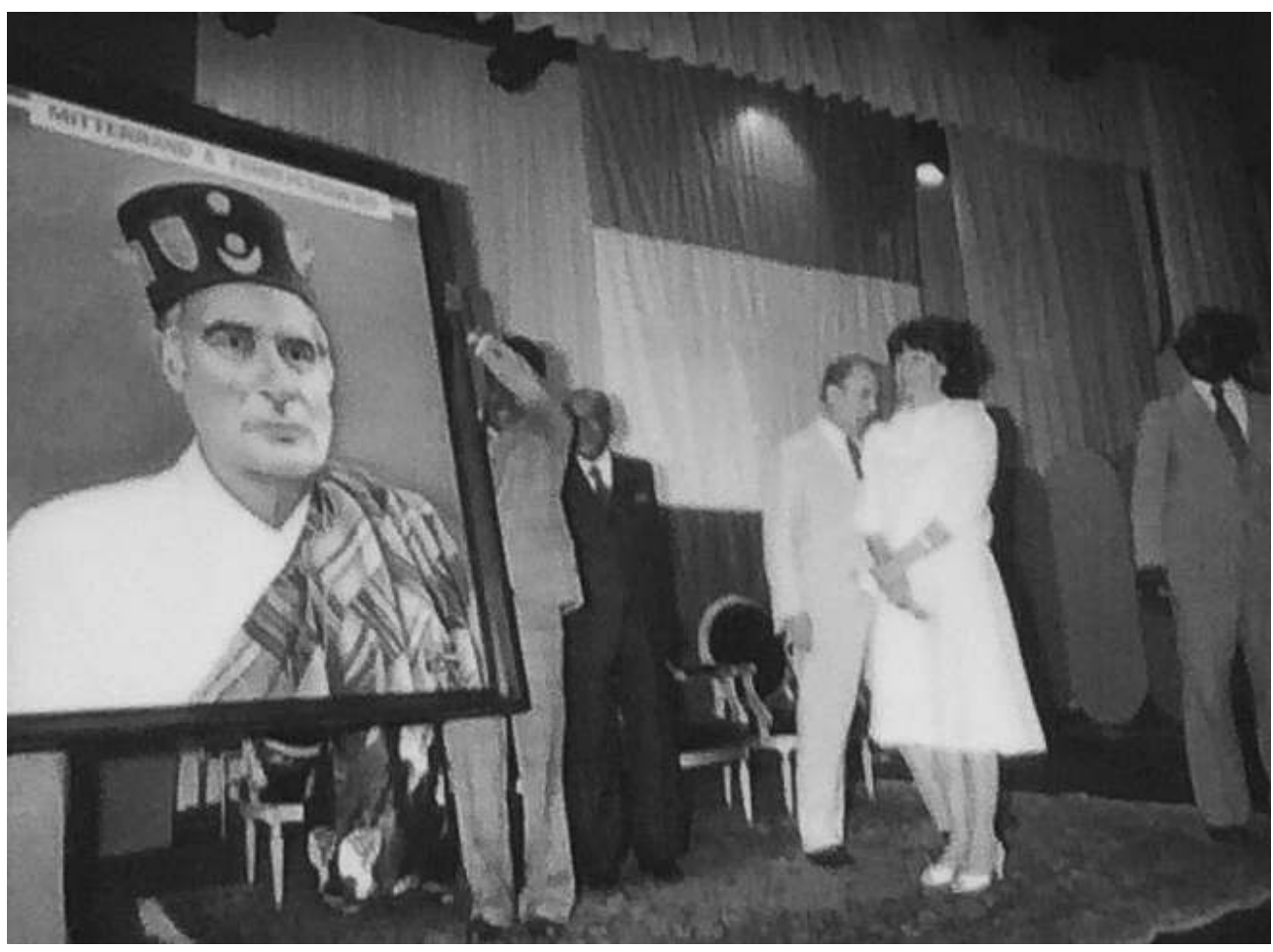

Voyage officiel de François Mitterrand en Côte d'Ivoire, remise de cadeaux à l'hôtel de ville de Yamoussoukro, 23 mai 1982, Archives nationales, AG/5(4)/SPH/7/P/2, reportage $n^{\circ} 4902$, planche $n^{\circ}$ 26 (détail)

(c) photographie de l'auteur

Au-delà des références personnelles, il est dès lors tentant de lire la récurrence de ce type de présents à travers un phénomène politique plus large, menant à l'incorporation des symboles des pouvoirs «traditionnels » dans le corpus de l'imagerie nationale véhiculée par les États postcoloniaux. Si les premières années d'indépendance virent, dans nombre de pays du continent africain, la marginalisation des détenteurs du pouvoir coutumier, dépeints par les nouvelles élites politiques comme les vestiges d'une époque à abolir, voire accusés de collusion avec les anciennes administrations coloniales, les années 1970-1980 marquèrent un retour de la figure du chef «traditionnel» sur la scène nationale ${ }^{31}$. Au Ghana, au Togo, en Guinée, plusieurs personnalités politiques tentèrent alors d'associer leur image à celle du souverain, dans un but de légitimation populaire. Le président ghanéen Kwame Nkrumah fait, à ce titre, figure d'exemple. En effet, l'art ashanti fut pour ce dernier une source d'alimentation privilégiée de son imagerie politique $^{32}$ : outre ses nombreuses apparitions à l'Assemblée ou aux Nations Unies en kente , le tissu traditionnellement associé à l'autorité du souverain ashanti, des objets d'art ashanti accompagnaient Nkrumah lors de certaines processions, tandis qu'un porteur d'épée et des ombrelles renforçaient l'association entre la figure du président et le pouvoir $\mathrm{du} \mathrm{roi}^{33}$. Le tissu kente constitua également un cadeau diplomatique de choix. Nkrumah offrit, par exemple, une pièce à son homologue libérien, William Tubman, en 1953 ou au Président tanzanien Julius Nyerere, photographié en 1962 drapé dans le kente reçu des mains du chef d'État ghanéen ${ }^{34}$. 
11 À l'instar de son voisin, Félix Houphouët-Boigny n'hésita pas non plus à déployer la symbolique et l'iconographie akan sur les supports de l'imagerie nationale ivoirienne. À l'occasion $\mathrm{du}$ huitième anniversaire de l'indépendance de la Côte d'Ivoire, l'administration émit un premier timbre représentant Le Sacrifice de la reine Abla Pokou qui, selon le récit fondateur baoulé, serait à l'origine de l'installation de ce peuple sur l'actuel territoire ivoirien ${ }^{35}$. D'autres éditions vinrent par la suite figurer les symboles de la famille royale akan ou l'intronisation d'un chef dans le district anyi. Cette promotion de l'identité culturelle akan à l'échelle nationale s'observe également dans l'organisation du voyage officiel de Valéry Giscard d'Estaing en Côte d'Ivoire, le président français étant accueilli, dès l'arrivée à l'aéroport d'Abidjan, par un cortège de chefs akan acheminés sur le tarmac dans une camionnette (fig. 6).

Fig. 6

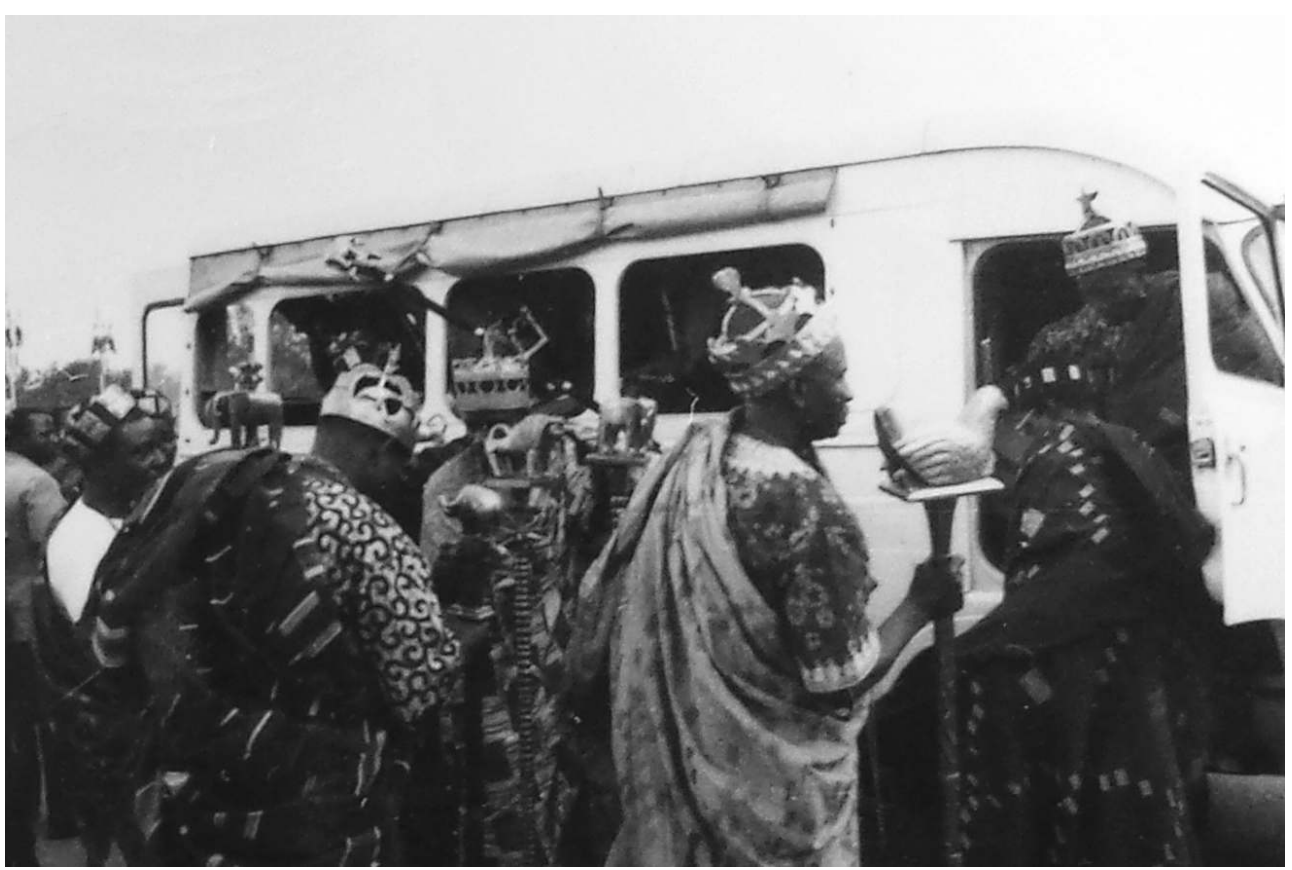

Voyage officiel de Valéry Giscard d'Estaing en Côte d'Ivoire, cortège de chefs akan, 11-15 janvier 1978, Archives nationales, $A G / 5(3) / 3506$, reportage n³733 (détail)

(c) photographie de l'auteur

\section{Du diplomatique à l'ethnographique : les tentatives de requalification du « cadeau » protocolaire}

Le 28 juin 1978, lors de la séance du Comité technique du musée des Arts africains et océaniens, $\mathrm{M}^{\mathrm{me}}$ Noll présenta devant l'assemblée présidée par $\mathrm{M}$. Landais et réunissant $\mathrm{M}$ me Riottot ainsi que MM. Daridan, Leiris et Lévi-Strauss les regalia akan reçus par Valéry Giscard d'Estaing à Yamoussoukro :

Mme Noll présente le don par le Président de la République de onze objets qui lui ont été offerts lors de son voyage en Côte d'Ivoire en Janvier 1978. Fabriqués pour la circonstance à Abengourou par des artisans ghanéens, ils constituent les attributs traditionnels des chefs coutumiers akan. L'ensemble se compose de trois cannes de porte-parole en bois doré pour deux d'entre-elles, plaqué de feuilles d'or pour la dernière, d'un chasse-mouche en bois doré, d'un sabre d'apparat, d'un siège de chef, 
en bois plaqué de feuilles d'or, d'une coiffure, de sandales, ainsi que de deux pendentifs en or $^{36}$. océaniens n'est pas anodine. Si Valéry Giscard d'Estaing fut éreinté par Le Canard enchaîné et Le Monde à propos de l'opacité de la gestion des cadeaux reçus dans le cadre de ses fonctions politiques ${ }^{37}$, il consentit ponctuellement - à l'image du Général de Gaulle ou de Georges Pompidou - à des donations au profit d'institutions muséales. La Revue du Louvre et des musées de France fait ainsi état de quatorze donations de cadeaux effectuées entre 1973 et 1979 au musée des Monuments français, au musée Guimet, au musée des Arts et Traditions populaires, au musée des Arts africains et océaniens, au musée de Blérancourt et au Louvre. D'autres présents, potentiellement perçus comme de moindre intérêt, furent déposés au Mobilier national, dès avant 1979 et l'affaire dite «des diamants de Bokassa », et ce jusqu'en mai $1981^{38}$. Le choix de la présidence de déposer les insignes akan au musée des Arts africains et océaniens pourrait ainsi suggérer la reconnaissance de la valeur tant plastique que culturelle de ces objets. Une telle démarche d'orientation de présents africains vers des institutions chargées de présenter les cultures africaines n'est pas isolée. Ainsi, la tenue d'apparat offerte en 1911 par l'empereur éthiopien Ménélik II à Victor-Emmanuel III fut déposée au musée Pigorini et codée comme « artefact ethnographique ${ }^{39}$ ». Cette transformation du cadeau diplomatique en un objet censé témoigner des traditions artistiques et culturelles de la société du donateur eut également lieu au musée de l'Homme, dépositaire des présents que le président Raymond Poincaré s'était vu offrir par la «mission abyssine». Présentée au public lors de l'exposition Éthiopie d'aujourd'hui. La terre et les hommes (avril-septembre 1975, musée de l'Homme), une partie des éléments composant un costume d'apparat éthiopien intégrèrent le parcours et le catalogue non comme témoignage de la longue histoire diplomatique unissant l'Éthiopie à l'Europe, mais en tant qu'illustration de la technologie éthiopienne en matière d'armement ou d'habillement, aux côtés d'autres artefacts conservés dans les collections de l'institution ${ }^{40}$.

De façon analogue, l'entrée en collection des regalia akan de Valéry Giscard d'Estaing semble avoir suscité une analyse particulière de ces objets, moins interprétés au regard de leur contexte de remise et des enjeux diplomatiques que celui-ci peut soulever, qu'en tant qu'images de la culture matérielle akan. Ainsi Étienne Féau interrogea-t-il son informateur, Charles Bieth ${ }^{41}$, sur «[...] les origines ethniques de ces sculpteurs, avec les noms si possible; pour quels chefs baoulé ou Agny ils travaillent d'ordinaire, à quelles occasions, avec quels matériaux, etc... ; sur le symbolisme enfin de ces objets ${ }^{42}$ ». Quelques mois après leur intégration aux collections du musée, une analyse scientifique des objets fut également réalisée par le laboratoire de recherche des musées de France afin d'étudier la composition du placage recouvrant les objets, comme pour s'assurer de la valeur de ces présents voire de leur authenticitée ${ }^{43}$. Le dossier d'œuvres contient, enfin, une note bibliographique manuscrite renvoyant vers des articles ou ouvrages de Doran H. Ross et Bohumil Holas, respectivement spécialistes des arts du Ghana et de la Côte d'Ivoire ${ }^{44}$. Cette volonté de documenter les objets à l'aune d'une littérature classique sur les productions akan vient alors en complément des observations de Holas, évoquées précédemment, sur les origines, la technique de fabrication, la symbolique ou l'usage des regalia en contexte traditionnel. 


\section{La « camelote » du président : la valeur des cadeaux selon Michel Leiris et Le Canard enchaîné}

15 Si les regalia de Valéry Giscard d'Estaing semblent avoir suscité l'intérêt en amont - de la part des présidents en personne - et en aval - à travers le travail de documentation initié par Étienne Féau - de leur intégration aux collections françaises, ce processus de patrimonialisation et de requalification des cadeaux ivoiriens n'eut pas lieu sans soulever quelques réticences, dont les fondements sont révélateurs de la délicate valorisation des présents africains en France. Ainsi, le compte rendu de la séance du 28 juin 1978 du Comité technique, au cours de laquelle fut présentée la donation présidentielle, met en lumière les sentiments ambigus de l'institution à l'égard de ces objets :

À la question de M. Leiris, de savoir s'il est protocolairement possible de refuser, le Directeur répond que cela lui paraît très difficile, les musées servant en l'occurrence de garde-meubles. On peut bien entendu mettre les objets en réserve quand ils ne sont pas exposables. Il serait en tout cas franchement inopportun de les mettre en dépôt dans un musée de province, lorsqu'ils ne sont pas dignes d'un musée national ${ }^{45}$.

16 La proposition, formulée à mots couverts par Michel Leiris et reprise par le directeur du musée, de refuser ces objets ou, à défaut, de les mettre au rebut dans les réserves d'un "musée de province », illustre la difficile intégration de ces objets dans le projet d'une institution qui, depuis 1960, avait achevé sa mutation en musée d'art. En effet, la décision prise par André Malraux de transformer l'ancien musée de la France d'outre-mer, frappé du sceau de l'idéologie coloniale, en institution dédiée à la mise en valeur des arts africains et océaniens, entraîna le repositionnement de sa politique d'exposition et d'acquisition, désormais fondée sur des «critères de qualité plastique et d'authenticité historique ${ }^{46} »$. De nombreuses pièces d'artisanat, autrefois exposées dans les vitrines du musée, furent écartées et, porté par des moyens financiers confortables, le musée privilégia un enrichissement des collections grâce à des acquisitions réalisées sur le marché de l'art ${ }^{47}$. Il est, par conséquent, aisé d'expliquer l'embarras du Comité technique face aux cadeaux de Valéry Giscard d'Estaing par l'inadéquation entre ces objets de commande, conçus spécifiquement pour être offerts au président français, et le nouveau projet culturel de l'institution.

Ce « malaise » du musée n'est pas sans entrer en résonance avec la façon dont, à la même époque, les cadeaux présidentiels africains furent décrits dans la presse. Si Le Canard enchainé, en révélant l'affaire des diamants, contribua à alimenter un débat, toujours vivant, sur la «valeur et la moralité du cadeau diplomatique (sans même plus porter sur son essence $)^{48}$ ", certains articles développèrent un discours inverse présentant les présents donnés par la présidence à des institutions culturelles comme des bricoles sans valeur. Ainsi, de façon à contrer les arguments de Valéry Giscard d'Estaing qui, pour prouver sa moralité et sa bonne foi, avait justifié le don de nombreux cadeaux à des musées, le journal dépouilla La Revue du Louvre et des musées de France dans le but de recenser l'ensemble des présents remis par le président depuis son accession au pouvoir. Parmi les quatorze interventions identifiées, Le Canard enchaîné, relève le don des regalia akan au musée des Arts africains et océaniens :

Don, par M. Valéry Giscard d'Estaing, de onze attributs de chefs coutumiers Akan

(Baoulé, Anyi, Attié) fabriqués à Abengourou par des artisans ghanéens, et de deux 
livres de B. Holas « Le séparatisme religieux en Afrique noire » et « Le Gagou, son portrait culturel ». Inv. MNAN 78-2-1 à 78-2-1149. en conservant les cadeaux les plus précieux - en l'espèce, les diamants offerts par Bokassa alors qu'il était encore ministre des Finances -, le journaliste entendait bien souligner le peu d'intérêt que représentaient ces pièces: "Conclusion offensante: de ses malles, si bien rangées à l'Élysée, et depuis cinq ans, le Président n'a sorti, pour en faire bénéficier les musées, que de la camelote sans valeur, ni marchande, ni artistique ${ }^{50}$.»

De Yamoussoukro à Paris, la trajectoire dessinée par les présents de Félix HouphouëtBoigny est à la fois atypique, par la richesse des points de vue et la diversité des personnalités que cet ensemble a polarisées, et représentative des difficultés rencontrées par les cadeaux africains une fois parvenus sur le sol français. Mis en regard des circonstances de donation, de la personnalité du donateur et du contexte politique, les insignes de pouvoir reçus par Valéry Giscard d'Estaing dévoilent leur dimension hautement polysémique. Incarnation de la richesse de la culture matérielle akan, symboles d'autorité par excellence, ces pièces se font également l'écho de la mise en scène de l'histoire du président Houphouët-Boigny et de l'appropriation d'un patrimoine local à l'échelle de l'État ivoirien. Cependant, leur intégration aux collections nationales en détermine des lectures plus restrictives. Malgré la volonté d'en faire des objets témoins des pratiques de pouvoir en pays akan, les réserves soulevées aussi bien par l'institution que par la presse montrent combien l'intérêt de ces cadeaux se laisse difficilement saisir par les schèmes de lecture habituellement utilisés pour jauger la valeur des artefacts africains.

\section{NOTES}

1. Jean-Pierre Bat, «Les diamants (de Bokassa) sont éternels. "Pré carré" et guerre fraîche : la fabrique de la Françafrique ", Afrique contemporaine, $\mathrm{n}^{\circ}$ 246, 2013, pp. 127-148.

2. La ville deviendra la capitale politique du pays à partir de 1983.

3. Programme de la visite officielle en Côte d'Ivoire du Président de la République et Madame Valéry Giscard d'Estaing, 11-15 janvier 1978, Archives nationales, AG/5(3)/3347.

4. Patrick Jarreau, « MM. Giscard d'Estaing et Houphouët-Boigny ont évoqué le problème du prix des matières premières ", Le Monde, 16 janvier 1978.

5. Idem, Ibidem.

6. Ces objets portent aujourd'hui les numéros d'inventaire 73.1978.2.1 à 73.1978.2.9 au sein des collections du musée du quai Branly - Jacques Chirac.

7. Lors de cette rencontre, Félix Houphouët-Boigny se vit ainsi offrir par son homologue une «coupe en jade avec un motif décoratif représentant une tête de bélier» de la maison Boucheron. Illustration de l'excellence de la joaillerie française, ce présent entendait également répondre aux goûts de son destinataire, connu par le protocole français pour posséder une « collection d'animaux en cristal de roche et pierres précieuses ». Proposition de cadeau pour le président de Côte d'Ivoire, Archives nationales, AG/5(3)/3461; Liste des cadeaux offerts lors du voyage officiel du Président en Côte d'Ivoire, Archives nationales, AG/5(3)/3462. 
8. Christaud M. Geary, The Voyage of King Njoya's Gift: A Beaded Sculpture from the Bamum Kingdom, Cameroon, in the National Museum of African Art, Washington, D.C., National Museum of African Art/ Smithsonian Institution, 1994, $60 \mathrm{p}$.

9. Mary Jo Arnoldi, « Gifts from the Queen: Two Malagasy Lamba Akotofahana at the Smithsonian Institution » dans Christine Mullen Kreamer et Sarah Fee (dir.), Objects as Envoys: Cloth, Imagery and Diplomacy in Madagascar, Washington, D.C., National Museum of African Art/Smithsonian Institution, 2002, pp. 95-120.

10. Dominique Jarrassé, «La qualification de l'objet. Leçon d'introduction au séminaire doctoral d'Histoire de l'art appliquée aux collections 2012-2013 », Les Cahiers de l'École du Louvre [En ligne], 2 | 2013, mis en ligne le 01 mars 2013, URL : http://journals.openedition.org/cel/524 ; DOI : $10.4000 /$ cel.524.

11. Étienne Féau, "L'art africain au musée des Arts d'Afrique et d'Océanie: collections et perspectives pour le musée du quai Branly », Cahiers d'études africaines, vol. 39, nº 155-156, 1999, pp. 923-938.

12. Compte-rendu de la séance du 28 juin 1978 du Comité technique du musée des Arts africains et océaniens, D003737/46590, Archives du musée du quai Branly - Jacques Chirac.

13. Lettre d'Étienne Féau au Docteur Bieth, Paris, le 24 mai 1978. Correspondance concernant la donation de la collection, D004785/47922, Archives du musée du quai Branly - Jacques Chirac.

14. Observations de M. Holas touchant les dons de la Présidence de la République, 26 mai 1978. Listes d'objets et notes diverses sur la collection 731978 2, D004785/47928, Archives du musée du quai Branly - Jacques Chirac.

15. Timothy F. Garrard, Gold of Africa. Jewellery and Ornaments from Ghana, Côte d'Ivoire, Mali and Senegal, the Collection of the Barbier-Mueller Museum, Munich, Prestel Verlag, 1989, 247 p.

16. Idem, Ibidem.

17. Georges Niangoran-Bouah, «Symboles institutionnels chez les Akan », L'Homme, 1973, vol. 13, $\mathrm{n}^{\circ}$ 1, pp. 207-233 ; Doran H. Ross, Gold of the Akan from the Glassel Collection, Houston, The Museum of Fine Arts, 2002, $303 \mathrm{p}$.

18. Idem, Ibidem.

19. Doran H. Ross, «The Verbal Art of Akan Linguist Staffs ", African Arts, vol. 16, $\mathrm{n}^{\circ} 1,1982$, pp. 56-67, 95-96.

20. Valéry Giscard d'Estaing, Le pouvoir et la vie, t. 2, Paris, Cie 12, 1991, p. 279 (c'est nous qui soulignons).

21. Félix Houphouët-Boigny, Mes premiers combats, Paris/Abidjan, Édition n ${ }^{\circ}$ 1/NEI, 1994, 125 p.

22. Note biographique de Monsieur Félix Houphouët-Boigny, Président de la République de Côte d'Ivoire, dossier "voyage officiel en Côte d'Ivoire (11-15 janvier 1978)», Archives nationales, AG/5(3)/3347.

23. V. Giscard d'Estaing, op. cit. note 20.

24. Idem, Ibidem.

25. Ces objets sont aujourd'hui conservés dans les collections du musée du Septennat, à ChâteauChinon (Nièvre), sous les numéros d'inventaire 58DFM/AFR0006 à 9.

26. Voyage officiel de François Mitterrand en Côte d'Ivoire, Archives nationales, AG/5(4)/SPH/7/ $\mathrm{P} / 2$, reportage $\mathrm{n}^{\circ} 4902$.

27. Cette œuvre de l'artiste K. Yssouf est également présentée au musée du Septennat et porte le numéro 58DFM/AFR0010.

28. Sur le contexte diplomatique de ce déplacement, voir Abou B. Bamba, «At the Edge of the Modern? Diplomacy, Public Relations, and Media Practices during Houphouët-Boigny's 1962 Visit to the U.S. ", Diplomacy \& Statecraft, vol. 22, n 2, 2011, pp. 219-238.

29. Memorandum for Mrs Evelyn Lincoln, Washington, May 21, 1962, John F. Kennedy Library and Museum, JFKPOK-023-003. 
30. Certaines de ces pièces auraient été présentées à Paris lors de l'exposition Corps sculptés, corps parés, corps masqués, chefs-d'œuvre de Côte d'Ivoire (18 octobre-15 décembre 1989, Paris, Grand Palais). Étienne Féau, « Exposition Côte d'Ivoire, Paris, Grand Palais, 15 oct.-15 dec. 1985 », dans Louis Perrois et Claude-François Baudez, Anthropologie de l'art: formes et significations (Arts de l'Afrique, de l'Amérique et du Pacifique), fascicule II, Paris, Orstom, 1989, pp. 5-12.

31. Claude-Hélène Perrot et François-Xavier Fauvelle-Aymar (dir), Le retour des rois: les autorités traditionnelles et l'État en Afrique contemporaine, Paris, Éditions Karthala, 2003, 568 p. ; C.-H. Perrot, «Les autorités traditionnelles et l'État moderne en Afrique subsaharienne au début du Xxi ${ }^{\mathrm{e}}$ siècle », Cadernos de Estudos Africanos, $n^{\circ}$ 16-17, 2009, pp. 15-33.

32. Janet Hess, "Exhibiting Ghana: Display, Documentary, and "National" Art in the Nkrumah Era ", African Studies Review, vol. 44, $\mathrm{n}^{\circ}$ 1, 2001, pp. 58-77; Janet Hess et Nii O. Quarcoopome, «Spectacular Nation: Nkrumahist Art and Resistance Iconography in the Ghanaian Independence Era [With Commentary] ", African Arts, vol. 39, n 1, 2006, pp. 16-25, 91-92.

33. J. Hess, art. cité note 32.

34. Cat. d'exp., Wrapped in Pride: Ghanaian Kente and African American Identity, sous la direction de Doran H. Ross, Los Angeles, UCLA Fowler Museum of Cultural History, 1998, 347 p.

35. Agbenyega Adedze, "Commemorating the Chief: The Politics of Postage Stamps in West Africa ", African Arts, vol. 37, $n^{\circ}$ 2, 2004, pp. 68-73.

36. Compte-rendu de la séance du 28 juin 1978 du Comité technique du musée des Arts africains et océaniens, D003737/46590, Archives du musée du quai Branly - Jacques Chirac.

37. Le 10 octobre 1979, Le Canard enchaîné ouvrait l'affaire dite «des diamants de Bokassa » en révélant qu'en avril 1973, lors d'un séjour en Centrafrique, Valéry Giscard d'Estaing, alors ministre des Finances, avait reçu une "plaquette [de diamants] de trente carats environ » des mains du dictateur Jean-Bedel Bokassa. Outre les implications politiques des révélations, largement relayées par les média - dont Le Monde au premier chef - et leur rôle dans la défaite du président aux élections de 1981, leur impact fut déterminant en France dans la perception des échanges de cadeaux comme pratique immorale. Voir, sur ce sujet, Alexandre GirardMuscagorry, Le cadeau postcolonial : les arts africains dans les échanges diplomatiques entre la France et les États de l'Afrique subsaharienne sous la $V^{e}$ République, mémoire de recherche, sous la direction de Dominique Jarrassé, Paris, École du Louvre, 2015, chap. 4.

38. Inventaire typographié des cadeaux diplomatiques remis au Mobilier National par la Présidence de la République entre 1978 et 1983, 2 janvier 2008, 16 p.

39. Benoit de L'Estoile, Le goût des Autres. De l'Exposition coloniale aux arts premiers, Paris, Flammarion, 2007, 453 p.

40. Cat. d'exp., Éthiopie d'aujourd'hui: la terre et les hommes, Paris, Laboratoire d'ethnologie, Muséum national d'histoire naturelle, 1975, pp. 70-73.

41. Ancien coopérant et professeur d'histoire-géographie, Charles Bieth créa en 1969 à Abengourou le "centre Bieth", à la fois centre social et école d'art, attaché à promouvoir le travail d'étudiants sans bagage académique. Voir Yacouba Konaté, «Arts and Social Dynamics in Côte d'Ivoire. The Position of Vohou-Vohou " dans Gitti Salami and Monica Blackmun Visona (dir.), A Companion to Modern African Art, Chichester, John Wiley \& Sons, 2013, pp. 371-388.

42. Lettre d'Étienne Féau au Docteur Bieth, Paris, le 24 mai 1978. Correspondance concernant la donation de la collection, D004785/47922, Archives du musée du quai Branly - Jacques Chirac.

43. Analyse de feuilles d'or plaquées sur 8 objets rituels, D004785/47928, Archives du musée du quai Branly - Jacques Chirac.

44. Listes d'objets et notes diverses sur la collection 73.1978.2, D004785/47926, Archives du musée du quai Branly - Jacques Chirac.

45. Compte-rendu de la séance du 28 juin 1978 du Comité technique du musée des Arts africains et océaniens, D003737/46590, Archives du musée du quai Branly - Jacques Chirac. 
46. Sandrine Treiner, Le musée des colonies: un espace de diffusion de l'idée coloniale en France, mémoire présenté en vue de l'obtention du Diplôme de maître d'histoire sous la direction de Françoise Raison, Université Paris 7, 1986, p. 118.

47. Jacqueline Eidelman, Anne Monjaret et Mélanie Roustan, MAAO: Mémoires, Paris, Marval, 2002, 163 p.

48. J.-P. Bat, art. cité note 1, p. 128.

49. C.-M. V., " Mensonges présidentiels. Les dons toquards d'Estaing ", Le Canard enchainé, 5 décembre 1979, p. 4.

50. Idem, Ibidem.

\section{RÉSUMÉS}

En janvier 1978, Valéry Giscard d'Estaing, alors en déplacement à Yamoussoukro dans le cadre d'un voyage présidentiel en Côte d'Ivoire, est élevé à la dignité de "Président honoraire de l'“Association des chefs coutumiers" " et reçoit, à cette occasion, plusieurs objets associés à l'exercice du pouvoir chez les peuples akan du pays. Donnés par la présidence française au musée des Arts africains et océaniens et aujourd'hui conservés dans les collections du musée du quai Branly - Jacques Chirac, ces présents diplomatiques sont atypiques, tant par leur originalité au regard $\mathrm{du}$ corpus très standardisé des cadeaux présidentiels africains, que par le nombre important de commentaires qu'ils ont suscités au cours de leur histoire. À l'aune de l'étude du contexte politique et diplomatique qui a vu naître ces objets, cet article entend mettre en lumière les motivations qui ont présidé à leur sélection, ainsi que leur réception mitigée en France sous l'influence du climat politique et institutionnel de l'époque.

In January 1978, Valéry Giscard d'Estaing, then visiting Yamoussoukro as part of a presidential visit to Côte d'Ivoire, was named Honorary President of the Association of Tribal Chiefs and received, on this occasion, several objects associated with the exercise of power among the Akan peoples of the country. Given by the French presidency to the Musée des Arts Africains et Océaniens and now in the collections of the Musée du Quai Branly-Jacques Chirac, these diplomatic gifts are atypical because of their originality compared to the very standardised corpus of African presidential gifts and the large number of comments they gave rise to throughout their history. In light of the study of the political and diplomatic context in which these objects were created, this article intends to shed light on the motivations that led to their selection, as well as their mixed reception in France under the influence of the political and institutional climate of the period.

\section{INDEX}

Mots-clés : cadeau diplomatique, échange de cadeaux, voyage officiel, protocole, art africain, Côte d'Ivoire, Akan, Baoulé, Valéry Giscard d'Estaing, Félix Houphouët-Boigny, musée du quai Branly, regalia

Keywords : State gift, gift exchange, State visit, protocol, African art, Ivory Coast, Akan, Baule, Valéry Giscard d'Estaing, Félix Houphouët-Boigny, musée du quai Branly, regalia 


\section{AUTEUR}

\section{ALEXANDRE GIRARD-MUSCAGORRY}

Élève conservateur du patrimoine à l'INP, Alexandre Girard-Muscagorry est diplômé du deuxième cycle de l'École du Louvre (parcours « recherche » en histoire de l'art) et de l'ESSEC Business School (Master in Science of Management). Il a soutenu en 2015 un mémoire de recherche, sous la direction de Dominique Jarrassé, consacré aux cadeaux diplomatiques offerts par les chefs d'État africains aux présidents français de la V $V^{\mathrm{e}}$ République. Il a enseigné de 2013 à 2015 à l'École du Louvre en tant que chargé de travaux dirigés en histoire des arts de l'Afrique subsaharienne.

$* * *$

Trainee museum curator at INP (National Institute of Cultural Heritage), Alexandre GirardMuscagorry is a graduate of École du Louvre (research branch, art history) and of ESSEC Business School (Master in Science of Management). He defended a research dissertation in 2015, supervised by Dominique Jarrassé, on diplomatic gifts from African heads of states to French presidents of the Fifth Republic. From 2013 to 2015, he taught at École du Louvre, supervising seminars in African art history. 